\title{
Treatment of Multiple Chalazions with Intralesional Kenalog-40 Injections in Juvenile Patient: A Case Report
}

\author{
Mirjana A. Janicijevic-Petrovic ${ }^{1}$, Snezana Jancic ${ }^{2}$, Katarina Janicijevic ${ }^{3}$, Andrijana Popovic ${ }^{3}$ \\ ${ }^{1}$ Clinic of Ophthalmology, Clinical Centre of Kragujevac, Kragujevac, Serbia; ${ }^{2}$ Clinic of Pathology, Clinical Centre of \\ Kragujevac, Kragujevac, Serbia; ${ }^{3}$ Faculty of Medical Sciences, University of Kragujevac, Kragujevac, Serbia
}

\begin{abstract}
Citation: Janicijevic-Petrovic MA, Jancic S, Janicijevic K, Popovic A. Treatment of Multiple Chalazions with Intralesional Kenalog-40 Injections in Juvenile Patient: A Case Report. OA Maced J Med Sci. 2013 Dec 15; 1(1):83-86 http://dx.doi.org/10.3889/oamjms.2013.016

Key words: chalazions; triamcinolone acetonide; Kenalog-40; incision-drainage; case report.

"Correspondence: Mirjana A. JanicijevicPetrovic. Clinic of Ophtalmology, Clinical Centre of Kragujevac, Zmaj Jovina 30, Kragujevac, of Kragujevac, Zmaj Jovina 30, Kragujevac,
Serbia. Mob: +38166013691. Fax: Serbia. Mo

+38134370073.

Received: 20-Aug-2013; Revised: 19-Oct2013; Accepted: 27-Oct-2013; Online first: 30-Oct-2013

Copyright: (c) 2013 Janicijevic-Petrovic MA. This is an open-access article distributed under the terms of the Creative Commons Attribution License, which permits unrestricted use, distribution, and reproduction in any medium, provided the original author and source are credited.

Competing Interests: The authors have declared that no competing interests exist.
\end{abstract}

\begin{abstract}
Aim: To case report the efficacy of subcutaneous steroid injections in the treatment of multiple chalazions and to evaluate the safety of intralesional injection in primary and recurrent chalazions in juvenile patient.

Case report: A 25-year-old man noticed lesions which appeared on upper lid during past three months. It was clinically presented with three focal, large and painful nodules in upper right eyelid. Inflammation process began on May the 1st of 2013. After one month in May 30th 2013 was directly administered synthetic steroid, Kenalog-40. Application of triamcinolone acetonide resulted in two satellite lesions, compared to the original, when the same incisions drained during the first surgery in July 5th 2013, with topical antibiotic-steroidal drugs and hot compresses. Second surgery was in July 26th 2013. Third surgery was performed in August 5st 2013 with second application of Kenalog-40. Combined treatment leads to recovery of clinical signs and inflammation of right upper eyelid with regressions of primary and recurrent chalazions.
\end{abstract}

Conclusion: Two steroid subcutaneous injections produced complete resolution of multiple primary and recurrent chalazions. Clinical imperative was that recurrent or unusual refractory chalazions require referral to Hystopathological examination of curetting to exclude malignancy.

\section{Introduction}

Chalazion is commonly benign as collection of chronic inflammatory lipogranulomatous tissue formed from meibomian glands which are lining the tarsus of the eyelid that is the site of pathology in a chalazion. Numerous meibomian glands located in the tarsus release an oily secretion that creates the precorneal tear film. Blepharitis or eyelid inflammation produces debris which can occlude meibomian gland orifices. There are two types of chalazion - external and internal, depending of localization in the eyelid. The main symptoms of chalazion include redness, swelling and swollen eyelids with painful and hard nodule [1].

Differential diagnosis of the eyelids pathology (for chalazion) include: pyogenic granuloma, lipogranuloma, papilloma of eyelid margin, focal blue nevus of eyelid, margin adenoid cystic carcinoma, margin sebaceous carcinoma, basso cell carcinoma, squamous cell carcinoma, etc [1].

Chalazions often resolve spontaneously or respond to the conservative therapeutic regimen. Incision-drainage with triamcinolone acetonide suspension intralesional injections and worm compresses may be applied at home to promote faster recovery [2].

Referral to ophthalmologists for incisiondrainage and triamcinolone acetonide suspension injections - synthetic steroid (Kenalog-40) is primary used because of potent anti-inflammatory effects in disorders of eye system) is warranted for recurrent or 
refractory chalazions. Dosage of Kenalog-40 is usually individualized, depending on the clinical pathology of eyelid and the patient's response to this treatment [2-8].

Internal hordeola may harden into chalazions, and need referral for use Erythromycin, Trimethoprim/Sulfamethoxazole, Clindamycin or Cephalexin for two weeks as known oral regimen and controversial of this therapeutic module $[9,10]$.

Malignancy must be ruled out in recurrent or refractory chalazions and because of that the tissue sample should be evaluated by the pathologists.

\section{Case report}

A 25-year-old male presented with complaint of three mounts history of right eyelid redness, swelling and mild pain.

Personal and family history of our young patient suggests underlying conditions such as seborrhea dermatitis, rosacea and blepharitis without the atopic and the other organ-system diseases. Myopia in our patient and his young brother was corrected with soft contact lenses during last 11 years (as risk factor for chalazions). Intraocular pressure bilaterally was $15-16 \mathrm{~mm} \mathrm{Hg}$ (by Goldman's tonometry) and visual acuity in both eyes was $20 / 20$ (by Snellen's test) remained stable before and after this treatment.

On examination, the superior portion of his right eyelid was erythematic and swollen, as well as warm and hard to palpation nodules (Figure 1).

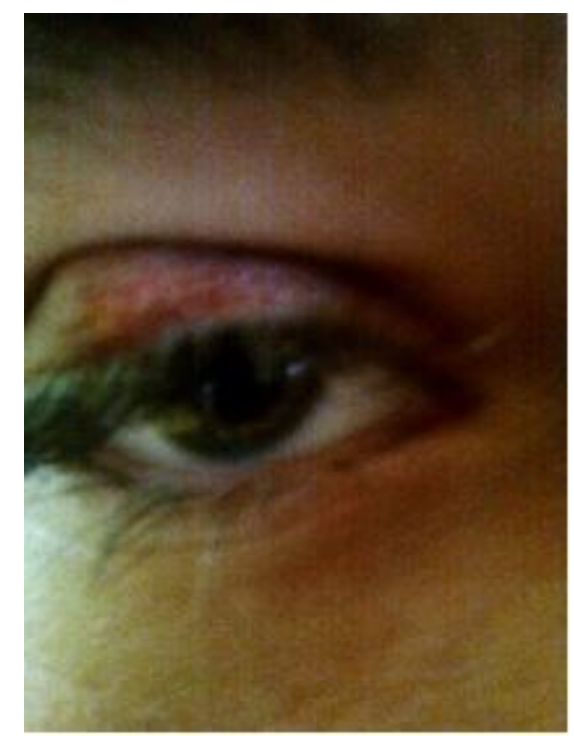

Figure 1: Superior right eyelid - redness, swollen, hard nodules and linear perilesional hypo-pigmentation caused by Kenalog-40.

There were no signs of consecutive conjunctivitis and scleritis. Inflammation process began in May $1^{\text {st }} 2013$, the disease started immediately and only after a few days of acute inflammation it expanded and closed whole upper right eyelid with pseudoptosis. It was followed by incision-drainage one month after the disease started. In May $30^{\text {th }} 2013$ was directly administered synthetic steroid, Kenalog-40 (first dose of $20 \mathrm{mg}$ ) that resulted in two new, satellite lesions, compared to the original. After the first surgery in July $5^{\text {th }} 2013$, when the content of chalazions was drained trough incision on the inside of the right upper eyelid, with administrated antibiotic-steroidal topical therapy (sol. Ofloxacin, sol. Neodexacyn, unguent Chloramphenicol - four time per day) and worm compresses. The second same surgical intervention was performed in July $26^{\text {th }} 2013$. Third surgical intervention was performed in August $5^{\text {st }} 2013$ with the second application of Kenalog-40 (doses of $20 \mathrm{mg}$ ) and Ofloxacin therapy in duration of two weeks. Described multi-combined treatment (with infiltrative xylocaine - anesthesia) leads to recovery of clinical signs and inflammation of the right upper eyelid and the regression of his chalazions. Authors have expressed positive experiences as an innovation in surgical treatment of chalazions with topical application of iodine solution in the final curettage through the incision (several times) which has resulted in lessening the signs of local and consecutive inflammation.

The material obtained by a complete excision during the surgery was sent to histopathologic analysis immediately after. Hystopathological findings pointed to dominant granular-inflammatory and cellular composition in favor of recurrent chalazions, absence of cellular malignancy and the other clinical entities. The nodule consists of many types of immune cells, including connective tissue macrophages such as histiocytes, multinucleate giant cells (Langhan's type), plasma cells and polymorphonuclear leukocytes (Figure 2).

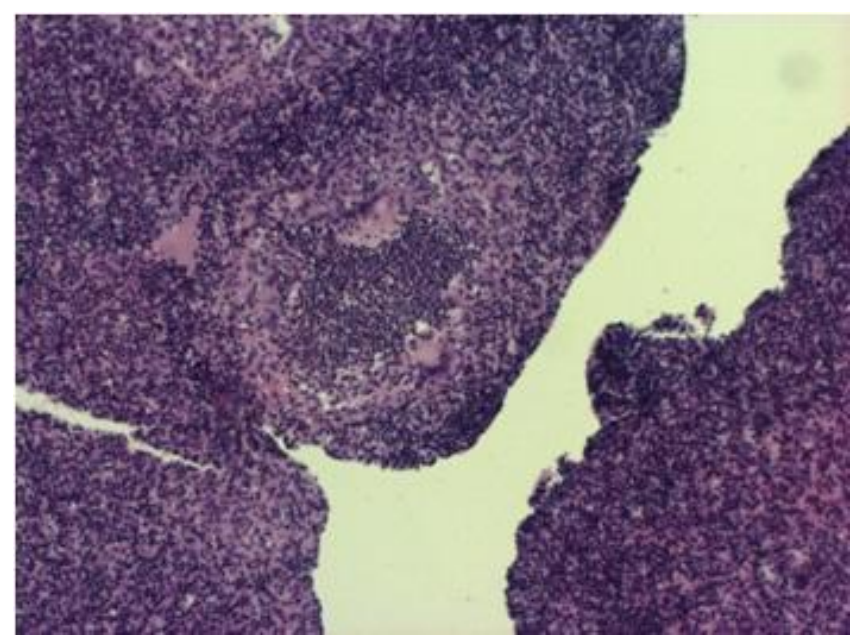

Figure 2: Hystopathological result: inflammatory granulomatous lesions (giant-cells, lymphocytes, plasma-cells, histiocytes and polymorphonuclear leukocytes.

There were no caused complications, such as subcutaneous atrophy, large skin depigmentation, skin necrosis, posterior subcapsular cataract and exophthalmos. The patient denied eye trauma, or eye 
and systemic diseases that could cause consecutive disease. Some of the signs can be the localized loss of eyelashes in area of his recurrent chalazions, and that can be sign for his malignancies in this affected area.

\section{Discussion}

Chronic inflammation leads to hyperkeratinization of ductal epithelium, which further obstructs the ducts. Chronic stagnation of meibomian secretion attracts bacteria that use lipids as nutritive substrate and releasing free fatty acids that can be toxic to the epithelium, which is well known biochemical process.

If chalazions don't resolve spontaneously, the instructions for patients is to apply a worm compress (3 to 5 minutes) to open the glands, then to digitally massages (either with fingers or cotton tips) the area to break and express the nodules, up to four times per day. If these procedures result to be ineffective, injections of triamcinolone acetonide (Kenalog-40) are to be applied directly into the chalazions. Approach the lesion from the palpebral side and inject 0.05 to $0.3 \mathrm{ml}$ in standard solution, using the insulin syringe. Two steroid injections produced a complete resolution of patient lesions - recurrent and refractory chalazions as the advantages in effective therapeutic module and without any drawbacks. The treatments for chalazions are incision and curettage technique (surgical extirpations) including removal of the pseudocapsule found surrounding the lesions and consists of a portion of the tarsal plate with fibroblastic scar tissue. Excision of the pseudocapsule, cosmetically cover a tumor and reducing the risk of re-accumulation of meibomian secretion in glands [1, 2].

Many studies compare three methods of chalazion treatment: first - intralesional triamcinolone acetonide injections, second - incision and curettage, third - combination of incision, curettage and intralesional triamcinolone acetonide injections. After these treatments some patients have complained on recurrence or development of new lesions; other patients had yellow-white deposits at the site of transcutaneous injections. Procedures were comfortable and safe. Intralesional corticosteroid injection is especially effective for young patients. Incision and curettage are recommended for patients with multiple inflammations of chalazions because it was showed to prevent recidivate. Combined incision, curettage and intralesional steroid injection is the most effective approach for patients with large, recurrent and multiple chalazions in regression of recidivate [2], what we applied and was showed as useful in case of our young patient.

Studies compare the outcome of intralesional corticosteroid injections and surgical treatment of chalazions. Intralesional steroid injections are effective and safe alternative to the other procedures for the treatment of chalazions. It is not associated with any serious complications, although skin depigmentation is relatively common in pigmented patients [3], which was not our case because our patient did not have dark skin.

Other studies have reported the cases of patients with linear perilesional hypo-pigmentation and atrophy developed after intralesional injections of steroids used for the treatment of the keloids. The linear perilesional hypo-pigmentation is a distinct complication after intralesional application of steroids and it is probably due to lymphogenous spread of the steroid suspension [4]. In our case authors noticed discreet linear perilesional hypo-pigmentation as only skin complication mentioned to the treatment.

Glucocorticoids are effective for the treatment of various inflammatory skin diseases, but their longterm use may lead to serious side effects such as skin atrophy. The Incidence of skin atrophies which following application of potent steroids is especially high in young patients. During last years the effects of Glucocorticoids on connective tissue have been clarified and the conclusion is that skin atrophy is mostly due to decrease in collagen synthesis. Collagen is the most contexture protein in the skin and the inhibition of its synthesis leads to skin atrophy. Many of studies explain the molecular mechanisms of steroid induced skin atrophy and validity of therapeutic possibilities [5]. We have not reviewed the cellular mechanisms of referred pathology because authors have not registered signs of eyelid skin atrophy.

Ben Simon et al were evaluating the efficacy of intralesional triamcinolone acetonide injection in primary and recurrent chalazions in patients. Success was defined as at least an $80 \%$ decrease in size with no recurrence. If the lesion recurred or regression was minimal, less than $50 \%$, further injections could be given as needed. If patients declined injection or did not respond to two or three injections must be referred for surgical excision and drainage. Most cases resolve with an average of one or two injections [6]. Triamcinolone acetonide may be considered as the first treatment in case where diagnosis is chalazion, which was confirmed in clinical case showed in this report.

Many authors as Ben Simon GJ compare treatment outcomes of intralesional triamcinolone acetonide injection with incision-curettage for primary chalazion performed under local anesthesia which results in optimal multi therapeutic results with no recidivate and no pain [7].

Norris $\mathrm{JH}$ says that the complete resolution was defined as lesion regression of $95-100 \%$. Triamcinolone acetonide injections may be considered as alternative first-line treatment in cases where diagnosis is clear and no biopsy is required [8], whose opinion we also share.

Chalazion surgery is a common minor 
surgical procedure used to treat internal chalazions with minor and infrequent complications. The results of many clinical studies suggest that the use of thermal cauterization with surgery has no impact on the recurrence rate of internal chalazions. Use of thermal cauterization should be left to the decision of ophthalmologist [11].

Many clinical studies are analyzing modules of therapy for chalazion, such as Kenalog-40 injection, surgical incision-curettage, and use of the worm and dry compresses $[12,13]$, also used in our case.

Bârsan $S$ et al have presented a clinical case of an infant with eye lid reconstruction after bilateral palpebral complete necrosis cussed by an extensive Pseudomonas aeruginosa infection. The substrate counterproductive evolution was identified by genetic analysis (X-linked Bruton-congenital agammaglobulinemia) [9], which by now did not appear in our professional practice.

Interesting study showed the efficacy and safety of subcutaneous Botulinum toxin A injections in the treatment of primary and recurrent chalazions with 2-5 IU in 0.2-0.5 $\mathrm{ml}$ of preserved saline solution. Lesion regression that did not respond to the average of two injections, would benefit more from surgical excision or systemic antibiotic therapy [14], but authors do not have experience in using Botulinum toxin $A$ in treatment of chalazions.

Some studies have isolated and identified bacterial pathogens causing ocular infections and determined their in-vitro antibacterial sensitivity to commonly used antibacterial agents. Staphylococcus aureus is frequently causes infections of eyelids and internal hordeola. Of all routinely used antibacterial tested, flouroquinolones (gatifloxacin and ofloxacin) represented the choice for treatment of chalazions $[10,15]$, as was used on patient from this case report.

As a conclusion, our patient with multiple chalazions was given intralesional injections of synthetic corticosteroid, Kenalog-40. Triamcinolone acetonide was given before and after surgical procedures that leads to success. Two steroid injections produced complete resolution of his recurrent and refractory lesions.

Despite the availability of new topical options, minor surgical intervention will unfortunately continue to play a role in the long-term management of the anterior segment problems.

The clinical imperative must be ruling out malignancy in recurrent and refractory chalazions and because of that the tissues sample should be evaluated by the pathologists.

\section{References}

1. Black RL, Terry JE. Treatment of chalazia with intralesional triamcinolone injection. J Am Optom Assoc. 1990; 61(12):904-6.
2. Mustafa TA, Oriafage $\mathrm{IH}$. Three methods of treatment of chalazia in children. Saudi Med J. 2001; 22(11):968-72.

3. Ahmad S, Baig MA, Khan MA, Khan IU, Janjua TA. Intralesional corticosteroid injection vs surgical treatment of chalazia in pigmented patients. J Coll Physicians Surg Pak. 2006; 16(1):42-4.

4. Friedman SJ, Butler DF, Pittelkow MR. Perilesional linear atrophy and hypo-pigmentation after intralesional corticosteroid therapy. Report of two cases and review of the literature. J Am Acad Dermatol. 1988; 19(3):537-41.

5. Oikarinen A, Autio P. New aspects of the mechanism of corticosteroid-induced dermal atrophy. Clin Exp Dermatol 1991 Nov; 16(6):416-9.

6. Ben Simon GJ, Huang L, Nakra T, Schwarcz RM, McCann JD, Goldberg RA. Intralesional triamcinolone acetonide injection for primary and recurrent chalazia: is it really effective? Ophthalmology. 2005; 112(5):913-7.

7. Ben Simon GJ, Rosen N, Rosner M, Spierer A. Intralesional triamcinolone acetonide injection versus incision and curettage for primary chalazia: a prospective, randomized study. Am J Ophthalmol. 2011; 151(4):714-18.

8. Norris $\mathrm{JH}$. Intralesional triamcinolone acetonide injection versus incision and curettage for primary chalazia: a prospective, randomized study. Am J Ophthalmol. 2012; 153(5):1005-6.

9. Bârsan S, Georgescu A, Stefăniu C. Eye lid reconstruction after bilateral palpebral complete necrosis after an extensive Pseudomonas aeruginosa infection (infected neonatal hordeola). Oftalmologia. 2010; 54(3):66-71.

10. Lindsley K, Nichols JJ, Dickersin K. Interventions for acute internal hordeola. Cochrane Database Syst Rev. 2013; 4:CD007742 [Pub Med - in process].

11. Sendrowski DP, Maher JF. Thermal cautery after chalazion surgery and its effect on recurrence rates. Optom Vis Sci. 2000; 77(11):605-7.

12. Brown TM, Pandya VB, Masselos K, Wang LW, Lee KJ, Figueira EC, Francis IC. A prospective randomized treatment study comparing three treatment options for chalazia: triamcinolone acetonide injections, incision and curettage and treatment with hot compresses: comment. Clin Experiment Ophthalmol. 2008; 36(4):394-5.

13. Ionova TA. The use of the preparation Kenalog- 40 for treating chalazion in children. Oftalmol Zh. 1989; (7):441-2.

14. Knezevic T, Ivekovic R, Astalos JP, Novak Laus K, Mandic Z, Matejcic A. Botulinum toxin A injection for primary and recurrent chalazia. Graefes Arch Clin Exp Ophthalmol. 2009; 247(6):789-94.

15. Ramesh S, Ramakrishnan R, Bharathi MJ, Amuthan $M$, Viswanathan S. Prevalence of bacterial pathogens causing ocular infections in South India. Indian J Pathol Microbiol. 2010; 53(2):2816. 